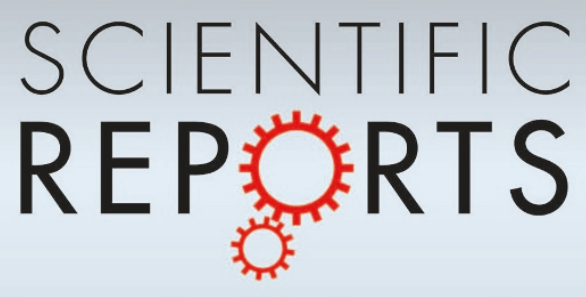

OPEN

SUBJECT AREAS:

APPLIED PHYSICS

FLUID DYNAMICS

INFORMATION THEORY AND COMPUTATION

MECHANICAL ENGINEERING

Received

15 November 2012

Accepted

22 May 2013

Published

25 June 2013

Correspondence and requests for materials should be addressed to J.W. (wangi@aps.anl. gov) or K.-S.I. (ksim@

Istc.com)

\section{Unraveling the Geometry Dependence of In-Nozzle Cavitation in High-Pressure Injectors}

\author{
Kyoung-Su Im ${ }^{1,2}$, Seong-Kyun Cheong ${ }^{1}$, Christopher F. Powell ${ }^{1}$, Ming-chia D. Lai ${ }^{3}$ \& Jin Wang ${ }^{1}$
}

\begin{abstract}
${ }^{1}$ Argonne National Laboratory, Argonne, IL 60439, USA, ${ }^{2}$ Livermore Software Technology Corporation, Livermore, CA 94551, USA, ${ }^{3}$ Wayne State University, Detroit, MI 48202, USA.
\end{abstract}

Cavitation is an intricate multiphase phenomenon that interplays with turbulence in fluid flows. It exhibits clear duality in characteristics, being both destructive and beneficial in our daily lives and industrial processes. Despite the multitude of occurrences of this phenomenon, highly dynamic and multiphase cavitating flows have not been fundamentally well understood in guiding the effort to harness the transient and localized power generated by this process. In a microscale, multiphase flow liquid injection system, we synergistically combined experiments using time-resolved $x$-radiography and a novel simulation method to reveal the relationship between the injector geometry and the in-nozzle cavitation quantitatively. We demonstrate that a slight alteration of the geometry on the micrometer scale can induce distinct laminar-like or cavitating flows, validating the multiphase computational fluid dynamics simulation. Furthermore, the simulation identifies a critical geometric parameter with which the high-speed flow undergoes an intriguing transition from non-cavitating to cavitating.

avitation can happen in any liquid flow and is present in various features depending on flow characteristics $^{1,2}$. For more than a century, cavitating flows have attracted researchers due to the damaging effects to fluid-handling devices, and the technological applications in the mechanical, chemical engineering, and biomedical fields ${ }^{1-10}$. On the one hand, cavitation causes stress erosion at liquid/solid interfaces ${ }^{3-8}$, ranging from minor damages over the span of long-time operations, to disastrous major damages in a relatively short period of time $^{3}$. On the other hand, it can also facilitate mixing in colloidal suspension, surface cleaning, improving heat and mass transfer ${ }^{3,4}$, and enhancing conventional chemical reactivity ${ }^{9,10}$. On micrometer scales, the sonoluminescence effect during the implosion of cavitation can generate plasma and ultrahigh temperature and facilitate unusual chemical reactions ${ }^{11-13}$. Moreover, one of the key effects of cavitation in microscale multiphase fluid flow is its association with strong turbulent flows. In the case of liquid fuel injection in internal combustion engines, the cavitating flow inside and outside of the injector nozzles dominates the breakup and atomization of the liquid fuel, critically affecting the combustion efficiency and emission ${ }^{14-23}$. Such a breakup process is driven by injecting liquid fuel through small orifices $(<100-\mu \mathrm{m}$ diameter $)$ at a high injection pressure $(>200 \mathrm{MPa})$ in advanced diesel engines ${ }^{20}$, thereby producing complex multiphase flows governed by many parameters such as turbulence and cavitation ${ }^{15-19}$. Among these is one of the most critical parameters: the cavitation induced by internal nozzle geometry ${ }^{21-26}$. Due to the paucity of direct and quantitative methods needed to visualize the transient multiphase flow phenomena in injection nozzles, a major approach to the problem has mostly been via modeling and simulation $^{21,22,24-26}$. In the meantime, experiments have been performed to demonstrate how the fluid dynamics inside and immediately outside the nozzle affects the jet breakup.

To this end, internal cavitating flows were visualized using scale-up optical nozzles ${ }^{19,27}$ and even replicas of realsize diesel nozzles made of transparent materials ${ }^{28,29}$ to demonstrate nozzle-geometry-induced cavitation and string cavitation due to interaction between flows in different orifices ${ }^{19,29}$. Even with a wealth of information from computational fluid dynamics (CFD) simulation and experiments, the quantitative data needed to correlate the nozzle geometry and the spray dynamics in an actual injection nozzle ${ }^{30}$ do not exist.

Recently, a time-resolved, synchrotron x-ray-based radiography technique has been developed for quantitatively probing the diesel fuel distribution outside the injector orifices with microsecond temporal and micrometer spatial resolutions ${ }^{30-36}$. In this work, we took advantage of the quantitative spray structure information revealed by the $\mathrm{x}$-radiography to investigate the influence of the internal geometry of a nozzle on the morphology of a highspeed liquid jet immediately after exiting the nozzle. We also developed a novel and effective numerical fluid 
dynamics simulation based upon the use of a simple yet realistic cavitation mode ${ }^{21-22}$ and an efficient algorithm ${ }^{37}$ for various orifice inlet geometries. In what follows, we demonstrate that the simulation of the internal flow field yields a remarkably good and quantitative agreement with the experiment data obtained immediately at the exit of the orifice. In a most direct and quantitative manner, the results reveal that the internal geometry variation, even on the micrometer scale, has a critical effect on the cavitation inside the nozzle and is responsible for the transition from a laminar to turbulent flow transition at this near-nozzle location.

\section{Results}

To illustrate the sole effect of the nozzle geometry, we chose two axisymmetric, single-orifice, high-pressure diesel nozzles with a subtle yet distinctive difference in feature: one had a rounded orifice inlet and the other had a sharp orifice inlet as shown schematically in Figs. 1(a) and (b), and denoted as R- and S-nozzles, respectively. At an injection pressure of $100-\mathrm{MPa}$, snapshots of the projected fuel mass distribution are shown in Figs. 1(c) to (h) at 99.4 (opening), 750 (quasi-steady), and $1369 \mu$ s (closing) after the start of injection in the near-nozzle region of the R-and S-nozzles, noting that the full needle valve lift was maintained between 500 and $900 \mu$ s and the valve was completely closed at $1450 \mu$ s (see Supplementary Materials). It is observed that the sprays were highly transient, especially during the opening and closing stages. Here, we use a parameter, spray angle $\theta$, to characterize the fuel distribution, as shown in Figs. $1 \mathrm{i}$ and $1 \mathrm{j} . \theta$ is quantitatively determined from the full-width-half-maximum of the distribution curves along the $y$-direction of the instantaneous mass distribution. During the nozzle opening and closing stages (99.4 and $1369 \mu \mathrm{s})$, the sprays from both nozzles showed similarly broad fuel distribution indicating higher-degree liquid breakup. In the quasisteady stage $(750 \mu \mathrm{s})$, the jet from the R-nozzle had minimum breakup (Fig. 1d) while that from the S-nozzle had a much broader spatial distribution (more breakup) in this near-nozzle region (Fig. 1g). $\theta$ remained as constants of $0.4^{\circ}$ and $3.7^{\circ}$ between 500 a
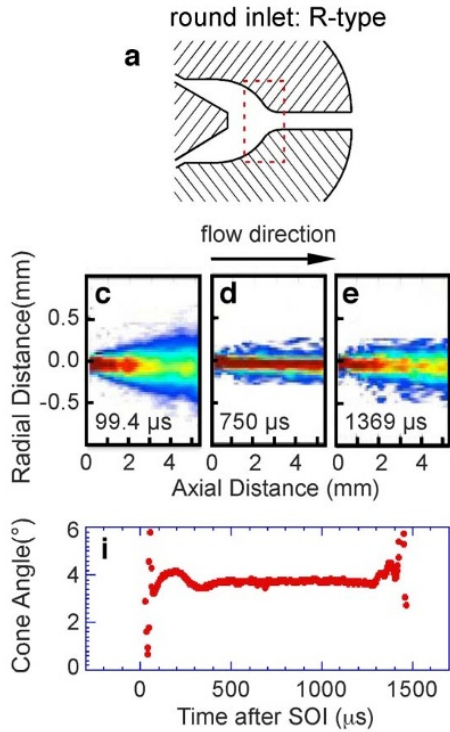

b
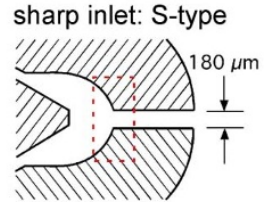

projected fuel density $\left(\mu \mathrm{g} / \mathrm{mm}^{2}\right)$
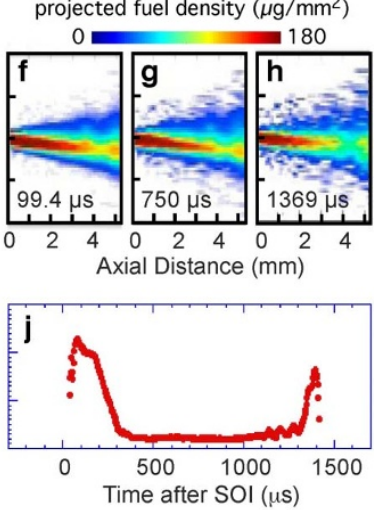

Figure 1 | Spray characteristics for two single-orifice nozzles: R-type and S-type. (a) and (b) are the schematics of the cross-sectional view of the two nozzles drawn to scale, $(\mathrm{c}-\mathrm{h})$ snapshots of time-resolved radiographs of the sprays with 1-ms nominal injection time and at $100 \mathrm{MPa}$ injection pressure, note the field of view was about $5 \mathrm{~mm}$ in the jet flow direction by $2 \mathrm{~mm}$ in the transverse direction, (i) and ( j) time-dependent microscopic spray angles with injection during the entire injection process of about $1.5 \mathrm{~ms}$. The sprays were stable between 500 and 1,000 $\mu$ s after the start of injection (SOI). and $1000 \mu$ s for R-and S-nozzles, respectively. We should emphasize here that the fuel mass distribution in the near-nozzle region was quantitatively determined to yield such a microscopic spray angle, different to the qualitative methods used in conventional visualization methods. The spray angle in this quasi-steady state, when the transient effect is minimized, is mostly induced by the internal geometry of the nozzles.

To further understand the breakup characteristics of the near nozzle sprays in the quasi-steady state, we obtained the fuel mass distributions along the $y$ - direction at $x=0.2,1.0$, and $3.0 \mathrm{~mm}$ as shown in Fig. 2 for both nozzles. Moreover, we performed a modeldependent 3-D reconstruction using the line-of-sight radiography data. The reconstruction models use the cross-sectional distribution of either a circular or an elliptical profile. The 3-D reconstructed results are represented by the liquid volume fraction (normalized by the measured specific gravity of the fuel: $880 \mu \mathrm{g} / \mathrm{mm}^{3}$ ). For the case of the jet from the R-nozzle, although the jet cross-sectional distribution distorted from a circular cross section at $0.2 \mathrm{~mm}$ to an elliptical shape at 1.0 and $3.0 \mathrm{~mm}$, the volume fractions of the fuel at the center of the spray core were close to unity, corresponding to $880 \mu \mathrm{g} / \mathrm{mm}^{3}$. Not surprisingly, the fuel mass density behaved considerably differently in the case of the S-nozzle jet. It quickly spread in the radial direction corresponding to a rapid decrease in the peak core volume fraction along the spray axis. At $3 \mathrm{~mm}$, the on-axis peak liquid volume fraction decreased to approximately $25 \%$. It should be noted that given the high spatial resolution in the liquid mass distribution, it is possible to map the degree of local breakup by referencing the liquid volume fraction. For example, while the liquid volume fraction was unity at the center of the spray $3.0 \mathrm{~mm}$ from the orifice
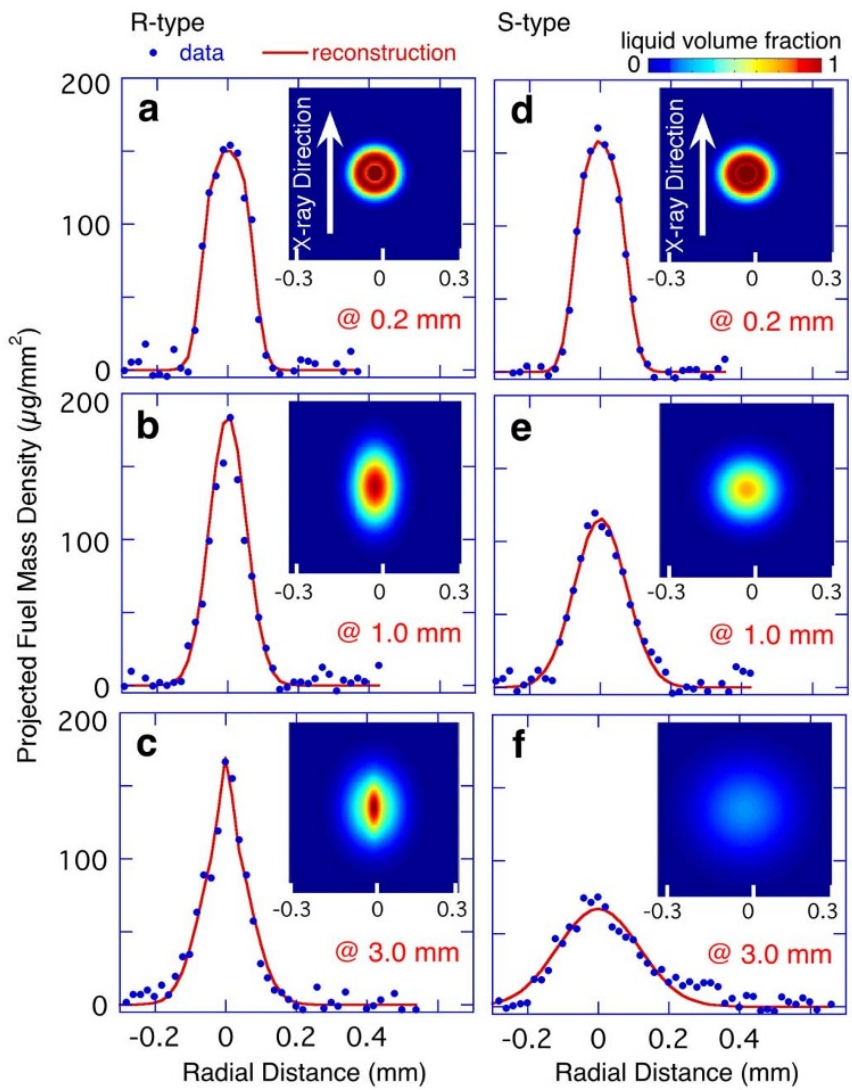

Figure $2 \mid$ Projected fuel mass density along the direction of the $x$-ray beam (measured by the time-resolved radiography) and the reconstructed 3D fuel density distribution at $0.2 \mathrm{~mm}, 1.0 \mathrm{~mm}$, and $3.0 \mathrm{~mm}$ based on the single projection data. $(\mathrm{a}-\mathrm{c})$ are for the R-type nozzle and $(\mathrm{d}-\mathrm{f})$ are for the S-type nozzle. 
exit, the low value away from the center marked a significant breakup resulting from the gas/liquid mixture that had a lower density than that of a liquid column, as observed in the R-nozzle case.

\section{Discussion}

The tomographic data revealed that the difference in the near-nozzle jet characteristics was so distinctive that it could be attributed to the liquid flow behavior inside the two nozzles with a subtle geometry difference at the orifice inlet. As suggested by a previous study ${ }^{38}$, it is widely believed that the different sprays are closely correlated with the internal cavitation. However, although $\mathrm{x}$-rays can visualize the inside of the nozzle, thus far it has been difficult to directly observe the highly transient and cavitating multiphase flows because of the thick steel housing that sustains high-pressure liquid. Hence, we resort the numerical simulation to understand such a complex multiphase problem, from the internal cavitation to the external liquid spray dynamics unveiled by the time-resolved $x$-radiography.

To achieve this we used the multiphase computational fluid dynamics (CFD) method to simulate the cavitating internal liquid flow based on the simple model and space-time conservation element and solution element that are described in both the Methods section and the Supplementary Materials. At the injection pressure of $100 \mathrm{MPa}$, the Reynolds number of the in-nozzle flow was on the order of $10^{4}$ and the flow was in the lamellar-to-turbulent transition region, if confined in a pipe. However, when the cavitating flow developed, the cavitation was a considerably more dominant and violent phenomenon than the turbulence ${ }^{21}$. Hence, in the quasisteady stage (500 to $1000 \mu$ s after start of injection), instead of using a turbulent model for the compressible fluid, we adopted a simple direct simulation technique by setting a sufficient number of relatively dense meshes $(0.9 \mu \mathrm{m}$ per mesh $)$ in the computational geometries, which were directly mapped from the nozzle drawings shown in Figs. 1(a) and (b). Parenthetically, we note that, there has not been an effective turbulence model developed for compressible flows.

The simulation results are shown in Figs. 3(a) and 3(b) as snapshots of the internal cavitating flows characterized by the gas phase void fractions. We immediately observed well-developed cavitation in the orifice of the S-nozzle, but only a much weaker gas void fraction in the R-nozzle. To the best of our knowledge, none of the current CFD methods are capable of modeling high-pressure sprays flowing continuously from the inside of the nozzle to the outside. Therefore, the external flow information cannot be readily obtained by simulation to compare directly with the experimental measurement in high injection pressure conditions at large Reynolds number in order to resolve the full dynamics characteristics of the liquid jets and sprays.

To overcome this difficulty, we derived a quantitative parameter, flow divergence angle, from the simulation results to describe innozzle flow up to the nozzle exit to infer the flow characteristics immediately outside the nozzle measured by the $\mu$ s x-ray radiograph. The flow divergence angle at a given location along the nozzle orifice is defined as the maximum ratio between the flow radial velocity component $\left(u_{y}\right)$ and that in the axial direction $\left(u_{x}\right)$. The location dependence of $u_{y} / u_{x}$ for both the R-nozzle and S-nozzle is shown as the insets to Figs. 3(a) and 3(b), respectively. With weak cavitation inside of the R-nozzle, the flow divergence angle value was close to zero (0.002). In the case of the S-nozzle, the value was more than one order of magnitude higher (0.04) close to the nozzle exit, corresponding to a virtue flow divergent angle of $4^{\circ}$ if the flow was not confined by the orifice, as in the case of the jet immediately outside of the nozzle. These angles agreed extremely well with the spray angle obtained by using time-resolved x-ray radiography. Details regarding the simulation of the development process for the cavitating flows in a form of the animation are included in the Supplementary Materials. This quantitative agreement between the simulation and
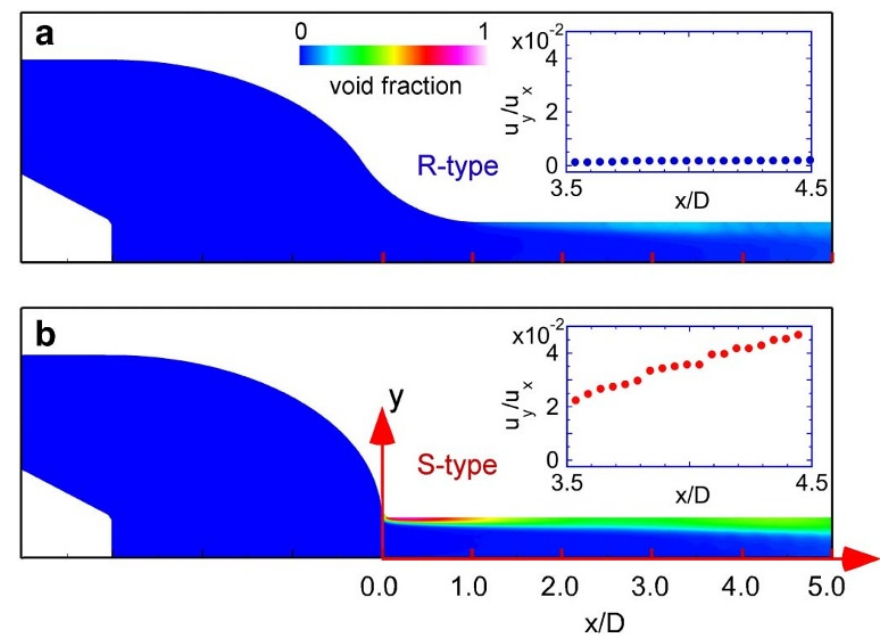

Figure 3 Simulation results of cavitating flows. (a) and (b) are the gas void fractions for the R- and S-type nozzles, respectively. The inserts depict the velocity ratio of radial to axial direction near the exit of the orifices. The axial coordinate is normalized by nozzle diameter, $D(180 \mu \mathrm{m})$, and its origin, $x / D=0$, is coincided with nozzle orifice inlet. At the orifice exit, the values of $x / D$ are 5 for both nozzles.

the measured data indicated that the spray morphology and breakup near the nozzle was strongly correlated with the cavitation inside the nozzle and the internal geometry of the nozzle, especially the orifice inlet responsible for the generation of the cavitation. When the internal flow has a radial velocity component, a radial expansion of the flow cannot happen due to the orifice confinement, resulting in a flow only in the axial direct. As soon as the confinement is removed at the nozzle exit, radial expansion at a small rate $\left(u_{y} / u_{x}\right)$ will happen immediately, as in the case for the S-nozzle. The R-nozzle provided a distinct contrast, the lamellar-like flow, with a minimum radial velocity component, which persisted to a few millimeters upon exit. Again, although this is a simplified approach, the spray angle represents the spray dynamics at the position immediately outside of the orifice exit. The correlation between the radial velocity components at both sides the orifice exit is best illustrated by the two simple nozzles operated at the same injection conditions but only with slight difference in their geometry.

As the simulation results agreed well with measurement, one of the critical questions that remained unanswered was how sensitive the cavitation generation was to the local structure. We note that it was extremely difficult, if not impossible, to micro-machine the orifice inlet with a controlled curvature with a resolution on the order of microns ${ }^{39,40}$. We took advantage of the realistic CFD simulation developed here to reveal the generation of the cavitation in the liquid flow inside the orifice to reveal the correlation between the cavitation and the curvature radius, $R$, of the orifice inlet, as shown in Fig. 4. Dimensionless $R$ is defined by the real curvature radius normalized by the orifice diameter $(D=180 \mu \mathrm{m})$, as shown in the inset of Fig. $4 \mathrm{a}$. We took a total of seven curvature values ranging from 0.15 to 0.75 with small increments in addition to the S-nozzle (0) and the Rnozzle (1.43). The internal flow divergence angle profiles near the nozzle exit for all the simulations using different $\mathrm{R}$ parameters are plotted in Fig. 4a. It is interesting to observe a sudden transition from the cavitating to the non-cavitating flow between $R=0.55$ and 0.6 , which is also highlighted in Fig. $4 \mathrm{~b}$, where the flow divergence angle values are captured at $x / D=4.4$. The simulation results of the gas volume fraction corresponding transition curvatures are shown in Figs. $4 \mathrm{c}$ and $4 \mathrm{~d}$, respectively, where the cavitation is readily observable when $R=0.55$ but not 0.6 . This "numerical experiment" clearly demonstrated that the cavitation was extremely sensitive to the change of the internal geometry near the orifice inlet, assuming that 




Figure $4 \mid$ Simulation results showing the correlation between the cavitation and the curvature radius, $R$, at the orifice entrance $(x / D=0)$. Dimensionless quantity $\mathrm{R}$, depicted in the inset to (a), was normalized by the nozzle diameter of $D$. (a) shows internal flow velocity ration (divergence angle), as a function of $R$. and (b) the values at $x / D=4.4$, indicated by the broken line in (a). A sudden drop of the value is observed clearly when $R$ increases from 0.55 to 0.6 . (c) and (d) are snapshots of the gas void volume fractions corresponding to the sudden transition from cavitating to noncavitating flows.

upstream flow conditions are identical. Although similar simulations on geometrical cavitation in nozzles have been attempted by many researchers ${ }^{23,24}$, the results here demonstrated the most direct and unambiguous correlation between the flow inside and outside of the orifice. Furthermore, in most real nozzles, due to the difficulties in the machining processes, the value of $R$ often varies across the orifice inlet circumference ${ }^{41}$. The ideal cases presented here pave the way to characterize and understand nozzles with a more complex geometry where cavitation can occur in a non-axisymmetric fashion.

To summarize, for the first time we used synergistically combined time-resolved $\mathrm{x}$-radiography data and multiphase computational fluid dynamics simulations to directly correlate the spray dynamics, breakup, and cavitation inside high-pressure injection nozzles. The quantitative agreement between the simulation and the experimental data revealed the origin of the cavitating flows generated by the nozzle geometry. The simulation further demonstrated that the geometrical dependence is so sensitive that a critical onset parameter exists at which the internal liquid flow underwent a cavitating to noncavitating transition. Most important, the near-nozzle jet breakup and spray formation was directly associated with the cavitating flows inside the nozzle. Technologically, the role of the geometry in the cavitation process can be used for optimizing high-pressure and high-speed liquid injection in internal combustion engines, for example, to simultaneously improve combustion efficiency and reduce the emissions.

\section{Methods}

The high-pressure common-rail diesel injection system and the test liquid fuel were identical to those described previously ${ }^{25-29}$. The fuel jet was injected into a spray chamber filled with helium, the lightest inert gas, at $0.1 \mathrm{MPa}$ and $28^{\circ} \mathrm{C}$ to minimize the liquid breakup due to the aerodynamic interaction between the fuel jet and the ambient gas $^{34}$. The molar mass of helium gas is less than $1 / 7$ of air, therefore exerting much lower stress than air would on the liquid jet, similar to the effect of a partial vacuum. The nominal injection pressure and duration were set to $100 \mathrm{MPa}$ and $1 \mathrm{~ms}$ in order to ensure a quasi-steady flow state in the middle of the injection when the internal flow was not disturbed by the motion of the injector needle valve. The motion of the needle valve, driven by a conventional solenoid mechanism, was monitored with ultrafast $x$-ray imaging (shown in the Supplementary Materials). From the onset of the needle valve opening to its fully closing, the measured injection time was $1450 \mu$ s while the needle valve was in a fully lifted (open) state between 500 and $900 \mu$ s from the start of injection. During the full needle lift stage (a time span of $400 \mu \mathrm{s}$ ), the sprays are considered to be in a quasi-steady state, which was later confirmed by the $\mathrm{x}$-ray radiographic images of the sprays. The transient characteristics of the injection will be discussed in detail in a future work. Here, we focus on the characteristics of sprays and internal flows in the quasi-steady state.

The microsecond radiographic data of the sprays (transmission) were collected by using a focused monochromatic (photon energy $E=8 \mathrm{keV}$ and bandwidth $\Delta E / E=$ $\left.10^{-4}\right) \mathrm{x}$-ray beam with a spot size of 200 (horizontal or jet axial direction, $\mathrm{x}$ ) by 30 (vertical or radial direction, y) $\mu \mathrm{m}^{2}$, at the 1-BM beamline of the Advanced Photon Source in conjunction with a fast avalanche photodiode point detector ${ }^{33}$. With monochromatic (single-wavelength) $\mathrm{x}$-rays being transmitted through a fluctuating liquid (in both space and time), the time evolution of the measured x-ray transmission could be simply related to the projected line-of-sight fuel mass distribution $[M(x, y, t)]$. From the measured $x$-ray transmission $I / I_{0}(x, y, t)$ and the fuel mass absorption coefficient $\left(\mu_{M}\right)$ of the attenuating material (fuel), the mass distribution can be evaluated as $M=-\mu_{M}^{-1} \ln \left(I / I_{0}\right)$. The radiographic results were highly quantitative and sensitive to $4 \mathrm{ng}$ of the fuel in the path of the $\mathrm{x}$-ray beam based on the signal-tonoise ratio of the data. In this case, the liquid fuel $\left(c a .880 \mathrm{~kg} / \mathrm{m}^{3}\right)$ was injected into a low-density medium (helium at $0.1 \mathrm{MPa}, 0.166 \mathrm{~kg} / \mathrm{m}^{3}$ ), the absorption of the $\mathrm{x}$-rays is solely by the liquid. Therefore, the liquid volume fraction can be determined quantitatively after $\mu_{M}$ is accurately determined with a liquid cylinder (unity liquid volume fraction) of known size ${ }^{31-36}$. This situation is different from a recent tomographic measurement of cavitating pipe flows using a commercial CT scanner ${ }^{42}$, where a polychromatic $x$-ray source was used and extensity calibration was needed to derive a three-dimensional (3-D) distribution of the approximate liquid volume fraction.

Caviating flow is a multiphase phenomenon where the gas phase can be generated in the liquid phase when the pressure drops below the vapor pressure. The fluid (liquid and gas) density can change from the liquid (ca. $880 \mathrm{~kg} / \mathrm{m}^{3}$ ) to vapor phase (ca. $1.2 \mathrm{~kg} / \mathrm{m}^{3}$ ) abruptly both temporally and spatially, which makes numerical simulation and modeling extremely difficult. Most importantly, a calculation model must incorporate the multiphase nature of the system and should be capable of dealing with compressible flows and handling of the rapid change of the speed of sound along with supersonic and subsonic flows due to complex local fluid compositions (see Supplementary Materials for details). A number approaches were utilized to model the complex cavitating flows, which can be classified in two major categories. The first one is a homogeneous equilibrium model using barotropic equation of state ${ }^{43,44}$. While earlier models used Rayleigh's equation ${ }^{45,46}$ along with incompressible liquid properties and mixture phases ${ }^{45-47}$, Schmidt et al. developed a fully compressible model of cavitation, extending the homogeneous equilibrium model to a compressible regime ${ }^{21}$. The second type of model has been developed based on more detailed physical processes involving bubble nucleation, growth, collapse, breakup, coalescence, and turbulent dispersion. In the present work, the main focus is to derive the effect of cavitation on the internal flow field and the orifice geometrical threshold at which the transition from lamellar to cavitation and turbulent flow occurs. While still far from an equilibrium state, the onset of cavitation can be primarily driven by local fluid pressure variations. Therefore, we chose the simple barotropic and compressible-fluid model $^{21}$ in our numerical simulation to compute the internal flow characteristics such as the fluid velocity distribution in the orifices. Despite this simplistic approach, the simulation yielded sufficiently accurate flow field characteristics that can directly compare with the ultrafast $\mathrm{x}$-radiography data on spray dynamics. Parenthetically, the limitation of the model is obvious - without consideration of cavitation dynamic processes, the density and the size distribution cannot be calculated or cannot be calculated as accurately as the more sophisticated models promise ${ }^{19,23,24,27,48}$

Meanwhile, we utilized the distinct advantages of the barotropic and compressiblefluid model to model the complex boundary conditions associated with cavitation flows, for instance the occurrence of local liquid/gas interfaces where shockwave conditions need to be taken into consideration, which plays an important role in fluid dynamics in the high-pressure, high-speed orifice flow case. The origin of the shockwaves is discussed in more detail in the Supplementary Materials. In short, the mixing of gas and liquid will significantly decrease the local sonic speed (to even $10 \mathrm{~s}$ $\mathrm{m} / \mathrm{s}$ ). To deal with the situation, Poinsot and Lele argued that the unsteady compressible calculation requires an accurate control of wave reflections from the outflow boundary to provide well-posedness into Navier-Stokes equations and proposed the Navier-Stokes characteristic boundary conditions ${ }^{49}$. In the original work ${ }^{21}$, the Navier-Stokes characteristic boundary condition method was used explicitly. In the present simulation, a simple and robust non-reflecting boundary condition was developed on the framework of the space-time flux conservation without specifying any characteristic wave ${ }^{50}$. On the other hand, the space-time conservation element and solution element method ${ }^{37}$ marked substantial improvement in both concept and methodology over traditional computational methods. By introducing non-overlapping conservation elements and a unique solution element of each conservation element's boundary as the tools for enforcing space-time flux conservation, a novel time marching, where space-time is staggered in the computational domain, is executed on the unified Euclidean space. As a result, fluxes at an interface can be evaluated without using any interpolation or extrapolation procedure, which in turn leads to the strong ability to capture shock waves and describe stiff boundaries without using Riemann solvers. More details about its nontraditional features are included in Supplementary Materials. Thanks to improvements to the previous applications ${ }^{36,51}$, the new simulation can deal with the high-speed liquid phase in addition to the relatively slow gaseous phase. Simple, accurate, and robust non-reflecting boundary conditions on the basis of the space-time flux conservation are employed without specifying any characteristic wave ${ }^{50}$. A detailed description on the simulation of the multiphase cavitating flow, numerical scheme, and treatment of the complex 
compressible boundary conditions based on space-time conservation element and solution element method is provided in the Supplementary Materials.

1. Brennen, C. B. Cavitation and Bubble Dynamics, Oxford University Press, New York, New York (1995).

2. Plesset, M. S. \& Prosperetti, A. Bubble dynamics and cavitation. Ann. Rev. Fluid Mech. 9, 145-185 (1977)

3. Arndt, R. E. A. Cavitation in fluid machinery and hydraulic structures. Ann. Rev. Fluid Mech. 13, 273-328 (1981).

4. Arndt, R. E. A. Cavitation in vortical flows. Ann. Rev. Fluid Mech. 34, 143-175 (2002).

5. Belahadji, B., Franc, J. P. \& Michel, J. M. A Statistical analysis of cavitation erosion pits. J. Fluids Eng. 113, 700-706 (1991).

6. Kato, H., Konno, A., Maeda, M. \& Yamaguchi, H. Possibility of quantitative prediction of cavitation erosion without model test. J. Fluids Eng. 113, 582-588 (1996).

7. Karimi, A. \& Leo, W. R. Phenomenological model for cavitation rate computation. Mat. Sci. Eng. 95, 1-14 (1987).

8. Reisman, G. E., Wang, Y.-C. \& Brennen, C. E. Observation of shock waves in cloud cavitation. J. Fluid Mech. 355, 255-283 (1998).

9. Kalumuck, K. M. \& Chahine, G. L. The Use of cavitating jets to oxidize organic compounds in water. J. Fluids Eng. 122, 465-470 (2000).

10. Gong, C. \& Hart, D. P. Ultrasound induced cavitation and sonochemical yields. J. Acoust. Soc. Am. 5, 1-8 (1998)

11. David, J. F. \& Kenneth, S. S. Plasma formation and temperature measurement during single-bubble cavitation. Nature 434, 52-55 (2005).

12. Bradley, P. B. \& Seth, J. P. Light scattering measurements of the repetitive supersonic implosion of a sonoluminescing bubble. Phys. Rev. Lett. 69, 3839-3842 (1992).

13. Didenko, Y. T., McNamara, III, W. B. \& Suslick, K. S. Effect of noble gases on sonoluminescence temperatures during multibubble cavitation. Phys. Rev. Lett. 84, 777-780 (2000).

14. Lefebvre, A. H. Atomization and Sprays, CRC Press (1988).

15. Reitz, R. D. Atomization and Other Breakup Regimes of a Liquid Jet, Ph.D Thesis, Princeton University, Princeton, NJ (1978).

16. Rietz, R. D. Modeling atomization processes in high-pressure vaporizing sprays Atomization Spray Technology 3, 309-337 (1987).

17. Kamimoto, T. Spray formation and combustion, in Advanced Combustion Science, Someya T. Ed, Springer-Verlag, Tokyo (1993)

18. Sou, A., Hosokawa, S. \& Tomiyama, A. Effects of cavitation in a nozzle on liquid jet atomization. Int. J. Heat Mass Transfer 50, 3575-3582 (2007).

19. Gavaises, M. \& Andriotis, A. Cavitation inside multi-hole injectors for large diesel engines and its effect on the near-nozzle spray structure. SAE Transactions. J. Engines 115, 634-647 (2006).

20. Lai, M.-C. \& Dingle, P. Diesel common rail and advanced fuel injection systems, SAE International, Warrendale, PA (2004).

21. Schmidt, D. P., Rutland, C. J. \& Corradini, M. L. A Fully compressible, twodimensional model of small, high-speed, cavitating nozzles. Atomisation Spray Technol. 9, 255-276 (1999).

22. Schmidt, D. P. \& Corradini, M. L. The Internal flow of diesel fuel injector nozzles: a Review. Int. J. Engine Res. 2, 1-22 (2001)

23. Arcoumanis, C. \& Gavaises, M. Linking nozzle flow with spray characteristics in a diesel fuel injector system. Atomization Sprays 8, 307-347 (1998).

24. Giannadakis, E., Gavaises, M. \& Arcoumanis, C. Modeling of cavitation in diesel injector nozzles. J. Fluid Mech. 616, 153-193 (2008).

25. Chen, Y. \& Heister, S. D. Modeling cavitating flows in diesel injectors. Atomization Sprays 6, 709-726 (1996).

26. Yuan, W. \& Schnerr, G. H. Numerical simulation of two-phase flow in injection nozzles: interaction of caviation and external jet formation. J. Fluids Eng. 125, 963-969 (2003).

27. Andriotis, A. \& Gavaises, M. Influence of vortex flow and cavitation on nearnozzle diesel spray dispersion angle. Atomization Sprays 19(3), 247-261 (2009)

28. Chaves, H., Knapp, M., Kubitzek, A., Obermeier, F. \& Schneider, T. Experimental study of cavitation in the nozzle hole of diesel injectors using transparent nozzles. SAE Technical Paper 95290 (1995).

29. Arcoumanis, C., Flora, H., Gavaises, M. \& Badami, M. Cavitation in real-size multi-hole diesel injector nozzles. SAE Technical Paper 2000-01-1249 (2000).

30. Lee, W.-K., Fezzaa, K. \& Wang, J. Metrology of steel micronozzles using x-ray propagation-based phase-enhanced microimaging. Appl. Phys. Lett. 87, 084105 (2005).

31. Cai, W. et al. Quantitative analysis of highly transient fuel sprays by time-resolved x-radiography. Appl. Phys. Lett. 83, 1671-1673 (2003).

32. MacPhee, A. et al. X-ray imaging of shock waves generated by high-pressure fuel sprays. Science 295, 1261-1263 (2002).
33. Powell, C. F., Yue, Y., Poola, R. \& Wang, J. Time-resolved measurements of supersonic fuel sprays using synchrotron x-rays. J. Synchrotron Rad. 7, 356-360 (2000).

34. Kastengren, A. L. et al. Nozzle geometry and injection duration effects on diesel sprays measured by x-ray radiography. J. Fluids Eng. 130, 041301 (2008).

35. Kastengren, A., Powell, C. F., Wang, Y.-J., Im, K.-S. \& Wang, J. X-ray radiography measurements of diesel spray structure at engine-like ambient density. Atomization Sprays 19, 1031-1044 (2009).

36. Im, K.-S. et al. Interaction between supersonic disintegrating liquid jets and their shock waves. Phys. Rev. Lett. 102, 074501 (2009).

37. Chang, S. C. The Method of Space-Time Conservation Element and Solution Element-A New approach for solving the Navier-Stokes and Euler equations. J. Comput. Phys. 119, 295-324 (1995).

38. Tamaki, N., Shimizu, M., Nishida, K. \& Hiroyasu, H., Effects of cavitation and internal flow on atomization of a liquid jet. Atomization Sprays 8, 179-197 (1998)

39. Jung, D. et al. Experimental investigation of abrasive flow machining effects on injector nozzle geometries, engine performance, and emissions in a diesel engine. Int. J. Automot. Technol. 9, 9-15 (2008).

40. Kent, J. C. \& Brown, G. M. Nozzle exit flow characteristics for square-edged and rounded inlet geometries. Combust. Sci. Technol. 30, 121-32 (1983).

41. Wu, Z. et al. Three-Dimensional Non-destructive Metrology of High-Pressure Injector Nozzles by High-energy X-ray Microtomography. (Submitted).

42. Bauer, D., Chaves, H. \& Arcoumanis, C. Measurements of void fraction distribution in cavitating pipe flow using X-ray CT. Meas. Sci. Technol. 23, 055302 (2012).

43. Delannoy, Y. \& Kueny, J. L. Two Phase flow approach in unsteady cavitation modelling cavitation and multiphase flow forum. ASME-FED 98, 153-158 (1990).

44. Ventikos, Y. \& Tzabiras, G. A Numerical method for the simulation of steady and unsteady cavitating flows. Comput Fluids 29, 63-88 (2000).

45. Kubota, A., Kato, H., Yamaguchis, H. \& Maeda, M. A. New modeling of cavitating flows: A Numerical study of unsteady cavitation on a hydrofoil section. J. Fluid Mech. 240, 59-96 (1992).

46. Yuan, W. \& Schnerr, G. H. Numerical simulation of two-phase flow in injection nozzles: Interaction of cavitation and external jet formation. J. Fluids Eng. 125, 963-969 (2003).

47. Chen, Y. \& Heister, S. D. Two-Phase modeling of cavitated Flows. Comput Fluids 24, 799-809 (1995)

48. Singhal, A. K., Athavale, M. M., Li, H. \& Jiang, Y. Mathematical basis and validation of the full cavitation model. J. Fluids Eng. 124, 617-624 (2002).

49. Poinsot, K. W. \& Lele, S. K. Boundary conditions for direct simulations of compressible viscous flows. J. Comput. Phys. 101, 104-129 (1992).

50. Chang, S. C., Himansu, A., Loh, C.-Y. \& Wang, X.-Y. Robust and simple nonreflecting boundary conditions for the Euler equations - A New approach based on the space-time CE/SE method. NASA/TM-2003-212495 (2003).

51. Liu, X. et al. Four Dimensional visualization of highly transient fuel sprays by microsecond quantitative x-ray tomography. Appl. Phys. Lett. 94, 084101 (2009).

\section{Acknowledgments}

We thank J. Schaller for providing the nozzles. Beamline staff at Sectors 1 and 7 of the Advanced Photon Source is acknowledged for the technical support. We are also grateful for the sponsorship of U.S. Department of Energy (DoE) Vehicle Technology Program. This work and the use of the APS were supported by the DoE, Office of Science, Office of Basic Energy Sciences, under contract No. DE-AC02-06CH11357. This work is also partially supported by Daegu Technopark, Korea, as part of Basic R\&D Supporting Program for Convergence Technology.

\section{Author contributions}

J.W. conceived the project. J.W., S.K.C. and C.F.P. designed the experiment and participated in data analysis. S.K.C., C.F.P. and K.S.I. conducted the experiment. K.S.I. and M.C.L. designed and performed the simulation and comparison to the experiment data. K.S.I. and J.W. wrote the manuscript. All authors discussed the results and reviewed manuscript.

\section{Additional information}

Supplementary information accompanies this paper at http://www.nature.com/ scientificreports

Competing financial interests: The authors declare no competing financial interests.

How to cite this article: Im, K.-S., Cheong, S.-K., Powell, C.F., Lai, M. \& Wang, J. Unraveling the Geometry Dependence of In-Nozzle Cavitation in High-Pressure Injectors. Sci. Rep. 3, 2067; DOI:10.1038/srep02067 (2013).

This work is licensed under a Creative Commons AttributionNonCommercial-NoDerivs 3.0 Unported license. To view a copy of this license, visit http://creativecommons.org/licenses/by-nc-nd/3.0 